\title{
Visualisation of plasmonic fields at the nanoscale with single molecule localisation microscopy
}

\author{
Christian Steuwe ${ }^{\mathrm{a}, \mathrm{b}}$, Miklos Erdelyi ${ }^{\mathrm{a}}$, G. Szekeres ${ }^{\mathrm{c}}$, M. Csete ${ }^{\mathrm{c}}$, Jeremy J Baumberg ${ }^{\mathrm{b}}$, Sumeet \\ Mahajan*d and Clemens F. Kaminski*a \\ ${ }^{a}$ Department of Chemical Engineering and Biotechnology, University of Cambridge, \\ New Museums Site, Pembroke Street, Cambridge, CB2 3RA, UK \\ ${ }^{b}$ Nanophotonics Centre, Cavendish Laboratory, University of Cambridge, \\ J J Thomson Avenue, Cambridge, CB3 OHE, UK \\ ${ }^{\mathrm{c}}$ Department of Optics and Quantum Electronics, University of Szeged, H-6720 Szeged, \\ Dóm tér 9, Hungary \\ ${ }^{d}$ Institute for Life Sciences, University of Southampton, University Road, \\ Southampton, SO17 1BJ, UK
}

Corresponding authors: SM (s.mahajan@soton.ac.uk),CFK( $f$ fk23@cam.ac.uk)

Plasmonic coupling of light to free electrons on metallic surfaces allows the confinement of electric fields far below the optical diffraction limit. Scattering processes of molecules placed into these plasmonic 'hotspots' are dramatically enhanced ${ }^{[1]}$ which is commonly used to increase the sensitivity of spectroscopic techniques for biological and chemical sensor applications ${ }^{[2,3]}$. Strikingly, hardly any measurement technique exists for the direct visualisation and characterisation of the underlying nanoscopic electromagnetic field distributions that either do not perturb the field ${ }^{[3,4]}$ or require complex electron beam imaging ${ }^{[5]}$. In this paper we introduce surface enhanced localisation microscopy (SELM), demonstrating the direct visualisation of fields on patterned plasmonic substrates using optical super resolution microscopy ${ }^{[6]}$. The observed strong photo-blinking behaviour of single molecules in plasmonic fields is exploited in SELM to map electromagnetic field distributions at nanometer resolutions.

Engineered nanostructured surfaces, which can sustain plasmon modes, are extremely important in technological applications such as enhancement of Raman scattering for detection of single molecules ${ }^{[7]}$ or to obtain reproducible characteristics for quantitative diagnostics ${ }^{[8]}$. The morphological characterisation of such nanostructures is generally obtained using scanning electron microscopy techniques ${ }^{[9]}$. Recently, using surface-attached photoactivatable fluorescent proteins morphological optical imaging of metallic nanostructures has also been shown to be possible [10] . Nonetheless, only a few techniques allow measuring electromagnetic field distributions of plasmonic modes with nanometer resolution ${ }^{[5,11]}$ without distortion and special or complex requirements such as photoactivable molecules. Therefore, finite and boundary element simulations are widely used to predict the field distribution on plasmonic surfaces, but this comes with significant uncertainties and limitations. Simulation results are algorithm dependent and can only predict fields for 'perfect' structures. In practice, however, structures free of imperfections do not exist ${ }^{[12]}$.

We demonstrate our technique to map nanoscopic EM field patterns in exemplar plasmonic structures, such as: Nanovoids, 'dish-like' structures with variable diameter $D^{[13]}$ (Figure $1 \mathrm{~A}$ ) and Klarite $^{\circledR}$, featuring a pyramidal pit structure as shown in Figure 1B. SEM images of both structures are shown in Figure 2A and B. Afterwards we compare experimental results with data obtained from

Nanoimaging and Nanospectroscopy III, edited by Prabhat Verma, Alexander Egner, Proc. of SPIE Vol. 9554, 95540Q · C 2015 SPIE · CCC code: 0277-786X/15/\$18 · doi: 10.1117/12.2190835 
Finite Element Modelling. Fluorescent molecules in contrast to Raman scatterers suffer from nonradiative electron transfer in the vicinity of metal surfaces ${ }^{[14]}$. Hence, the maximum fluorescence emission is observed at a distance away from the surface due to the competition between nonradiative decay and field enhancement. This distance was found to be $\sim 20 \mathrm{~nm}$ and kept by tagging a DNA strand at the 53rd base pair position above the surface (probe strand). This strands binds to a surface strand covalently attached to the metal. By controlling the concentration of the probe strand, the dye density on the surface was kept sparse enough for the localisation of individual fluorescent molecules.

A

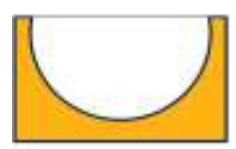

B

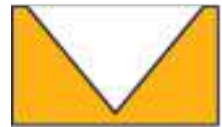

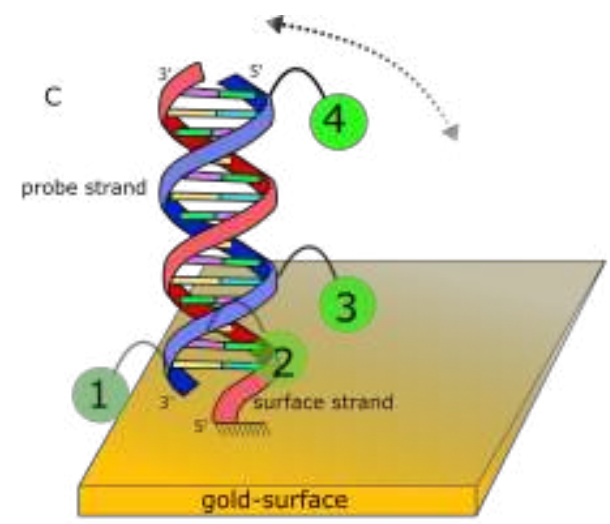

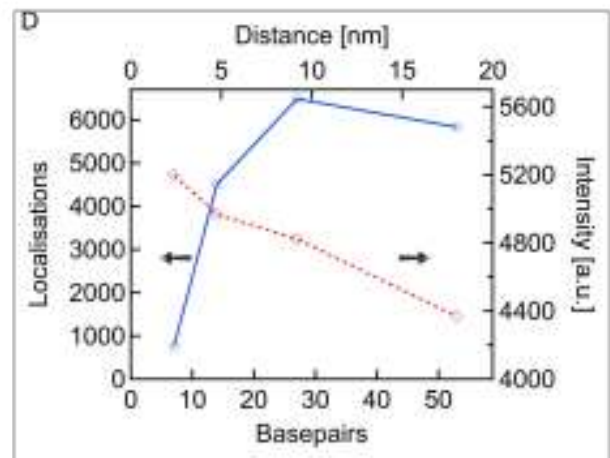

Figure 1A, B: Schematic of the nonovoid and Klarite ${ }^{\circledR}$ structured surfaces respectively. C: Sketch of the labelling technique involving two DNA strands. One attaches to the surface through a thiol, the second strand carries a dye molecule at the $5^{\prime}$ end. DNA swaying as possible cause of blinking. D) Localisations per 4200 frames vs. dye distance from the surface.

For single molecule localisation a subset of fluorophores is required to be active at any given time to ensure a minimal probability of overlap between their point spread functions in any one recorded image ${ }^{[15]}$. A large number of sparse fluorescence images and superimposing reconstructed molecule positions permits the structure of a labelled object to be resolved at nm precision ${ }^{[16]}$. For SELM on Klarite and nanovoid surfaces, image stacks of 3000 to 10000 frames with 20 ms integration time each were acquired. Strikingly, we observed very strong fluorescence blinking of the dye in the absence of any external agent such as a 'switching buffer', which is typically used in stochastic optical reconstruction microscopy_ ${ }^{[17]}$. A static gold background was removed effectively with a subtraction algorithm calculating a slowly converging median for improved localisation.

Superresolution imaging by single molecule localisation therefore becomes possible without requirement for any specific photactivation mechanisms, switching between fluorescent on- and offstates. The autonomous blinking behaviour was investigated by varying the distance of the dye molecule by using different complementary strands with labels at different positions as illustrated in figure $1 C$ and D. Image stacks of 4200 frames were processed for each case and the number of localisations (blinking events) plotted versus linker length as well as the mean intensity of all localisations in a stack. This graph demonstrates that it is the number of blinking events which decreases, rather than the intensity of each event. If a molecule is fluorescent; the fluorescence is as strong as permitted by the local electric field. The closer the fluorescent tag is to the surface, the lower the probability that it enters an on-state. This leads us to the assumption that the molecules 
sway on the surface due to Brownian motion, which has been observed previously ${ }^{[18]}$. The molecules' tilt angles change dynamically on the surface due to diffusion as per an elastic bending diffusion model developed earlier ${ }^{[19]}$. This dynamic bending enables two phenomena by movement of the dye. It changes the distance and hence the strength of the electric field and brings it closer or farther from the surface which would determine whether the fluorescence gets quenched or not. It is clear that the dye attached at the least distance from the surface will have the least change in its position, and will be quenched the most and therefore have the lowest number of blinking events. Although the quenching dominates the number of events in this case, the few times the fluorophore escapes the tunnelling (quenching) regime, high intensities due to surface enhanced fluorescence (SEF) are observed as the plasmon field is strongest near the surface.
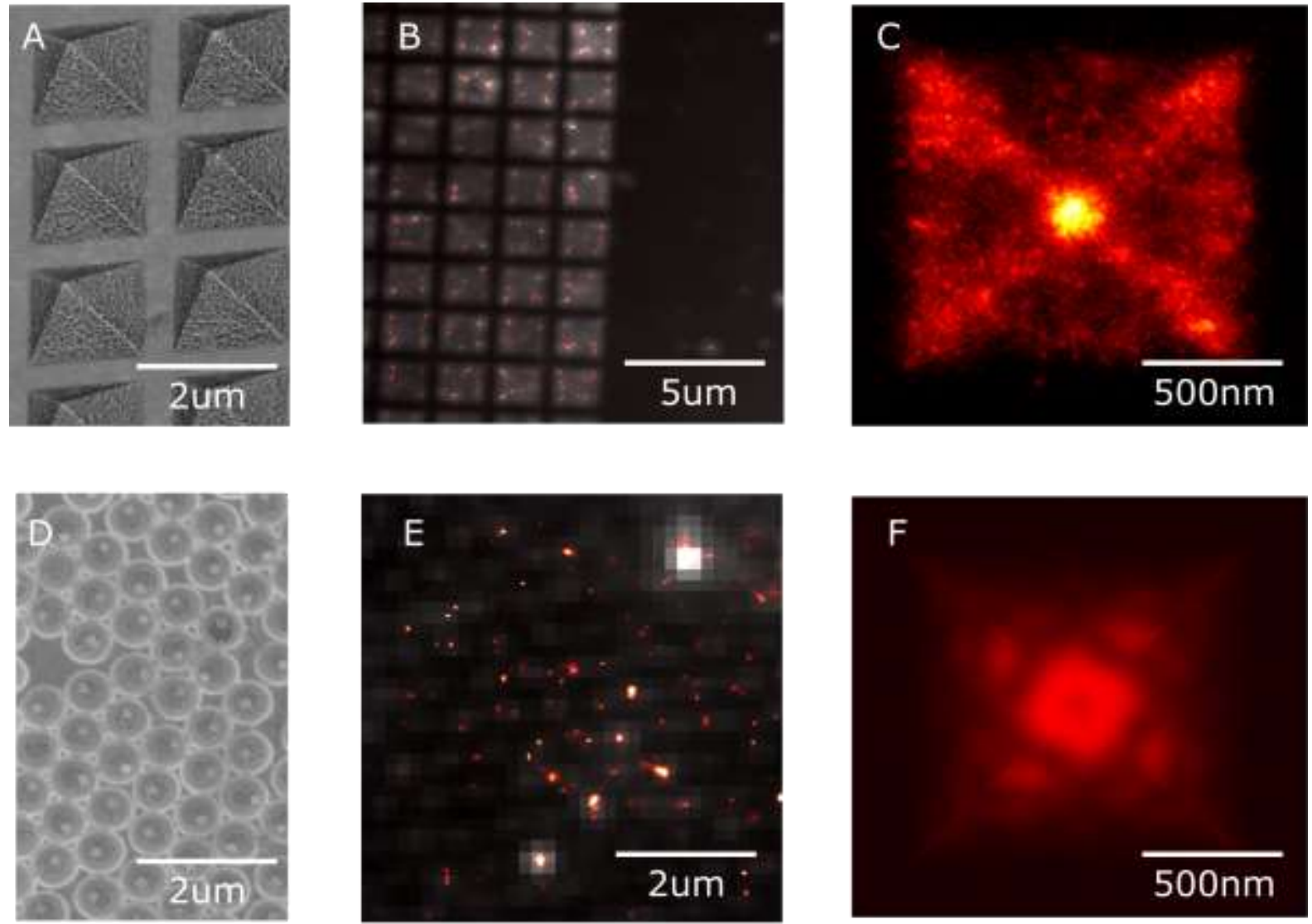

Figure 2 A: Scanning electron microscope picture Klarite pits (indicating their surface roughness). B: Reconstructed SELM image of a Klarite substrate. Unlike the diffraction limited bright field fluorescence image overlaid where only the periodical structure of Klarite is resolved, distinct features inside the pits become visible. C: Overlay of SELM reconstructions of all pits of $B$. The average localisation and intensity distribution of Klarite pits shows strong enhancement along the edges and towards the tip of the pit structure. The flat sides of the pit are less active. D: SEM images of a 600nm nanovoid surface. E: SELM images of 600nm nanovoids. F: Finite element simulations of the electric fields inside a Klarite pit.

Figure $2 \mathrm{~B}$ shows the reconstructed super resolution image of the surface enhanced fluorescence on Klarite. Clearly, features within individual pits are resolved while the conventional bright field image only shows coarse structures. Features observed inside the pits resemble one another across the 
surface demonstrating the high reproducibility of the Klarite substrate geometry from pit to pit. In a highly magnified view, however, fine distribution details of the generated surface enhanced fluorescence is revealed. This is due to variable roughness on the nanoscale (see Figure $2 \mathrm{~A}$ for an SEM image) along the edges of the pit. Surface roughness is a crucial parameter affecting plasmon generation and is likely to play an important role in determining the overall field enhancements that occur in Klarite ${ }^{[20]}$. This demonstrates the ultra-high sensitivity of SELM to visualise differences and stochastic variations of the field patterns from pit to pit. Plasmons are visualised as hotspots on SELM images which are representative of field enhancements, caused not only by the Klarite geometry but also and importantly due to surface defects. These features cannot be taken into account in simulations which do not address the random surface roughness. To compare electric field simulations with the SELM reconstructions, we averaged the experimentally acquired imaging data over many individual Klarite pits, to simulate the field distribution from an idealised 'smooth' Klarite pit. Such an overlay of images from 60 SELM images of individual pits shown in fig. $2 B$ is presented fig. 2C. Strong field modes are evident towards the tip of the pit structure, as well along the edges. This observation is highly reproducible. Moreover, the wide-field imaging ability of SELM allows capturing the interaction between different plasmonic structures, which in the case of Klarite confirms that there is no delocalisation of plasmons between the pits.

In contrast to Klarite, the super resolved SELM image of nanovoid structures in Figure 2E shows remarkable differences to Klarite. Individual hotspots are not well separated for void diameters of $\mathrm{D}=600 \mathrm{~nm}$ (fig. 2E ). Unlike Klarite, nanovoids are known to support delocalised plasmons propagating from one void to the next via the rim ${ }^{[21]}$. This ' $r i m^{\prime}$ ' mode has been shown to be involved

in generating SERS ${ }^{[21,22]}$. Hence, it is very likely that the localisation at the intersection of the rims of three neighbouring nanovoids, takes place. The intense bright regions or 'hotspots' observed between neighbouring voids, point to the high field strengths and highly confined plasmons which give rise to strong SEF. These images of patterned substrates such as Klarite and nanovoids demonstrate that the SELM is a generic method suitable for imaging plasmon fields in nanoscopic detail without resorting to complex photoactivation methods.

\section{Conclusion}

Surface enhanced localisation microscopy can overcome conventional, diffraction limited far field scattering techniques to infer field patterns of the nanoscopic plasmons. The direct visualisation of field patterns and details within active plasmonic areas using single molecule localisation superresolution microscopy combined with surface enhanced fluorescence is presented. This technique even allows investigating smallest plasmonic hotspots arising from surface imperfections.

Furthermore, the direct switching of molecules near the surface is feasible without the application of reducing agents. This opens new possibilities for uncomplicated super-resolution microscopy of metallic nanostructures useful in a wide range of fields including plasmonics, surface-enhanced spectroscopies, electrochemistry and surface-science.

\section{References}

[1] M. Fleischmann, P. Hendra, A. McQuillan, Chemical Physics Letters 1974, 26, 163.

[2] J. Homola, S. S. Yee, G. Gauglitz, Sensors and Actuators B: Chemical 1999, 54, 3; R. F. Aroca, Phys. Chem. Chem. Phys. 2013, 15, 5355.

[3] W. L. Barnes, A. Dereux, T. W. Ebbesen, Nature 2003, 424, 824. 
[4] A. V. Zayats, I. I. Smolyaninov, A. A. Maradudin, Physics reports 2005, 408, 131.

[5] J. Nelayah, M. Kociak, O. Stephan, F. J. Garcia de Abajo, M. Tence, L. Henrard, D. Taverna, I. Pastoriza-Santos, L. M. Liz-Marzan, C. Colliex, Nat Phys 2007, 3, 348.

[6] M. J. Rust, M. Bates, X. Zhuang, Nature methods 2006, 3, 793; M. Heilemann, S. van de Linde, M. Schüttpelz, R. Kasper, B. Seefeldt, A. Mukherjee, P. Tinnefeld, M. Sauer, Angewandte Chemie International Edition 2008, 47, 6172.

[7] K. Kneipp, Y. Wang, H. Kneipp, L. T. Perelman, I. Itzkan, R. R. Dasari, M. S. Feld, Physical review letters 1997, 78, 1667.

[8] R. J. Brown, M. J. Milton, Journal of Raman Spectroscopy 2008, 39, 1313.

[9] J. Goldstein, D. E. Newbury, D. C. Joy, C. E. Lyman, P. Echlin, E. Lifshin, L. Sawyer, J. R. Michael, Scanning electron microscopy and X-ray microanalysis, Springer, 2003.

[10] H. Lin, S. P. Centeno, L. Su, B. Kenens, S. Rocha, M. Sliwa, J. Hofkens, H. Uji-i, ChemPhysChem 2012, 13, 973.

[11] C. E. Hofmann, E. J. R. Vesseur, L. A. Sweatlock, H. J. Lezec, F. J. García de Abajo, A. Polman, H. A. Atwater, Nano letters 2007, 7, 3612.

[12] W. Barnes, Journal of Optics A: Pure and Applied Optics 2009, 11, 114002.

[13] J. J. Baumberg, T. A. Kelf, Y. Sugawara, S. Cintra, M. E. Abdelsalam, P. N. Bartlett, A. E. Russell, Nano letters 2005, 5, 2262.

[14] J. R. Lakowicz, Analytical biochemistry 2001, 298, 1; P. Anger, P. Bharadwaj, L. Novotny, Physical review letters 2006, 96, 113002.

[15] E. J. Rees, M. Erdelyi, D. Pinotsi, A. Knight, D. Metcalf, C. F. Kaminski, Optical Nanoscopy 2012, 1, 1.

[16] E. J. Rees, M. Erdelyi, G. S. K. Schierle, A. Knight, C. F. Kaminski, Journal of Optics 2013, 15, 094012.

[17] S. van de Linde, R. Kasper, M. Heilemann, M. Sauer, Applied Physics B 2008, 93, 725.

[18] V. Chan, D. J. Graves, P. Fortina, S. E. McKenzie, Langmuir 1997, 13, 320; U. Rant, K. Arinaga, S. Fujita, N. Yokoyama, G. Abstreiter, M. Tornow, Nano Letters 2004, 4, 2441.

[19] A. Anne, C. Demaille, Journal of the American Chemical Society 2006, 128, 542.

[20] J. Gersten, A. Nitzan, The Journal of Chemical Physics 2008, 73, 3023.

[21] R. M. Cole, J. J. Baumberg, F. J. García de Abajo, S. Mahajan, M. Abdelsalam, P. N. Bartlett, Nano Lett. 2007, 7, 2094.

[22] S. Mahajan, M. Abdelsalam, Y. Suguwara, S. Cintra, A. Russell, J. Baumberg, P. Bartlett, Physical Chemistry Chemical Physics 2007, 9, 104. 